\title{
Article
}

\section{'We don't have recipes; we just have loads of ingredients': explanations of evidence and clinical decision making by speech and language therapists}

McCurtin, Arlene and Carter, Bernie

Available at http://clok.uclan.ac.uk/12760/

McCurtin, Arlene and Carter, Bernie ORCID: 0000-0001-5226-9878 (2014) 'We don't have recipes; we just have loads of ingredients': explanations of evidence and clinical decision making by speech and language therapists. Journal of Evaluation in Clinical Practice. ISSN 1356-1294

It is advisable to refer to the publisher's version if you intend to cite from the work. http://dx.doi.org/10.1111/jep.12285

For more information about UCLan's research in this area go to http://www.uclan.ac.uk/researchgroups/ and search for <name of research Group>.

For information about Research generally at UCLan please go to http://www.uclan.ac.uk/research/

All outputs in CLoK are protected by Intellectual Property Rights law, including Copyright law. Copyright, IPR and Moral Rights for the works on this site are retained by the individual authors and/or other copyright owners. Terms and conditions for use of this material are defined in the policies page. 


\title{
We don't have recipes; we just have loads of ingredients: Explanations of evidence and clinical decision-making by speech $\&$ language therapists
}

\begin{abstract}
Introduction Research findings consistently suggest that speech and language therapists (SLTs) are failing to draw effectively on research-based evidence to guide clinical practice. Such decision-making is one of the least visible aspects of practice. This study aimed to examine what constitutes the reasoning provided by SLTs for treatment choices and whether science plays a part in those decisions.

Method This study, based in Ireland, reports on the qualitative phase of a mixed methods study which examined attitudes underpinning treatment choices and the therapy process. SLTs were recruited from community, hospital, and disability work settings via SLT mangers who acted as gatekeepers. A total of three focus groups were run. Questions were developed based on the original research aims and from the four key domains - client suitability, clinical experience, training and the role of science $=-$ generated from survey data from a previous phase.. Data were transcribed, anonymised and analysed using thematic analysis.

Results In total,48 participants took part in the focus groups. The majority of participants were female, represented senior grades and had basic professional qualifications. Three key themes were identified: practice imperfect; practice as grounded and growing; and critical practice. Findings show that treatment decisions are 'scaffolded', primarily on practiceevidence. This practice-evidence is both routine and evolving and decisions are both habitual and engaged. The uniqueness of each patient results in dynamic and pragmatic practice, constraining the application of unmodified therapies.

Conclusion The findings reflect the complexities and paradoxes of clinical practice as described by SLTs. Practice is pivoted on both the patient and clinician, through their membership of groups and as individuals. The findings suggest that scientific thinking is a component of decision-making; a tool with which to approach the various ingredients and the dynamic nature of clinical practice. However, these scientific elements do not necessarily reflect EBP as typically constructed.
\end{abstract}

\section{Introduction}

Research findings consistently suggest that speech and language therapists (SLTs) fail to draw effectively on research based evidence to guide clinical practice (Brener et al, 2003; Nail-Chiwetalu \& Bernstein-Ratner, 2007; O’Connor \& Pettigrew, 2009; Zipoli \& Kennedy, 2005). Yet little is really understood about why this might be or whether problems with the uptake of research-evidence reflect problems with its scientific nature. Proponents of evidence-based practice (EBP) argue that EBP is the best model to act as a foundation for and guide clinicians' treatment decisions, although these arguments seldom take into account the complexities and realities of clinical practice. Clinical decisions are increasingly subject to public analysis and, as Richardson, Higgs \& Abrandt-Dalhgren 
(2004:13) comment, 'insights into the foundations on which our practice is based can help us respond to the glare of scrutiny in contemporary health care'.

In this paper we explore insights into these foundations through examining SLTs reasoning for treatment choices and interpretations of science in practice.

\section{Evidence-based practice (EBP) and science}

Most definitions of EBP (Dollaghan, 2007; Sackett, 2006) represent a commitment to incorporating science into practice and clearly highlight three pillars: research; clinical experience; and patient preferences. EBP is seen as an attempt to introduce more objectivity (Ioannidis \& Lau, 2000), as a process for facilitating clinicians to arrive at well-reasoned decisions (Gambrill, 2005) and making them more 'rational' (Jenicek, 2006). However, numerous studies consistently suggest, that EBP has been met with less than keenness by SLTs (Brenner et al., 2003; Nail-Chiwetalu \& Bernstein-Ratner, 2007; O'Connor \& Pettigrew, 2009; Zipoli \& Kennedy, 2005). This is despite a high level of investment in research dissemination (Walsh \& Rundall, 2001) thus provoking questions of why such an apparently reasonable - and scientific - model is struggling to find a place in clinical practice.

The use of research however, is only one example of science in practice. Sagan (1996:22) for example, maintains that science is not merely a body of knowledge but a way of thinking, arguing that the scientific method 'is far more important than the findings of science'. Scientific practice is thus evident in behaviours such as experimentation (Roulstone, 2001), generating hypotheses (Shermer, 2001), questioning (Profetto-McGrath, 2005), argumentation (Jenicek, 2006) and problem solving (Klahr, 2000). What underlies scientific behaviour can be encompassed by the notion of critical thinking and the use of an organised, structured and scientific approach rather than one which is characterised by randomness and unsubstantiated guesswork (Kida, 2006). In essence, the scientific method should enable clinicians to confidently defend their work and the quality of care a profession offers (Richardson, Higgs \& Abrandt-Dahlgren, 2004).

\section{Therapy and Reasoning}

Whereas science is seen as precise, controlled and objective, clinical practice has been described as being swampy lowlands (Schon, 1983) and grey-zoned (Naylor, 1995). This swamp exists because the range of clinical decisions, questions and forms of uncertainty are numerous (Ely et al. 1999) and because clinicians face 'ill-defined problems' (Higgs \& Jones, 2000:4). Translating these multiple considerations into reasoned clinical decisions is complicated. Clinical practice, by its nature, requires constant engagement and adaptation and is highly dependent on clinicians' capacity to reason. Despite the importance of clinical reasoning in SLT practice-decisions, the SLT literature on the subject suffers by comparison with its allied disciplines (McAllister \& Rose, 2000) and it is currently unclear what supports intervention decisions and whether they are scientifically grounded. Practice-evidence, essentially knowledge originating from clinical practice (Higgs et al., 2001) is known to inform SLT decision-making. Such knowledge is often interpreted as 
subjective, biased and prone to error (Jones, Grimmer, Edwards, Higgs \& Trede, 2006; Kahmi, 2004) and given the uniqueness of clinical experience, Tonelli (2010) argues, it will necessarily lead to variability. Research suggests that SLTs value practice knowledge highly, being guided more by their experience and the opinions of colleagues (Bennett et al., 2003; O’Connor \& Pettigrew, 2009 Zipoli \& Kennedy, 2005) than scientific sources. Practice knowledge also originates from continuing education (e.g. Law and McColl, 1989) which may serve to influence the use of less scientific practices such as Watson and Lof (2004) found for non-speech oral motor exercises (NSOMEs). Thus, there continue to be questions around what practice-evidence constitutes and EBP has done little to help define or explain.

Patient perspectives are one of the three pillars supporting intervention decisions in the EBP model. Although the weighting on patient opinion is not new (Gilliam \& Gilliam, 2006), its integration into treatment decisions is not necessarily routine. Iacono and Cameron (2009) found SLTs on the whole to be using a directive approach. While client factors do appear to be strong determinants in treatment choice as indicated by SLTs in Joffe and Pring's (2008) study, these tend to be patient characteristics such as client age and cognitive abilities. Studies suggest that SLTs may be more inclined to incorporate patients and families as therapy assistants or technicians than engage them as partners in the process (Iacono and Cameron 2009, Watts-Pappas, McLeod, McAllister and McKinnon 2008). For example, Watts-Pappas et al (2008) established that although SLTs involved parents in service delivery, involvement in service planning was less frequent. Beecham (2004) argues that although the ideal is the incorporation of patient preferences, it may not be as practically possible as it is conceptually ideal. Moreover, explicit information on exactly how to integrate client preferences is still relatively absent in the literature (Entwistle \& O'Donnell, 2001).

While Kahmi (1999) comments that clinicians have been educated to make informed decisions, decision-making is one of the least visible aspects of practice. The reasons SLTs have for choosing using the therapies they do may reflect philosophies of practice such as functionality (Kahmi, 2006a; Papadopoulos, Noyes, Barns, Jones \& Thorn, 2012).

Mackenzie et al (2010) for example, found that SLTs aimed for simplicity and function in their treatment choices. Scientific constructs such as theory may not be seen as useful when selecting treatments as seen in Joffe \& Pring's (2008) study. Instead, Kahmi's (1999:93) 'because it works' reasoning may explain many treatment decisions; similar findings are evident in both discipline specific and allied discipline studies (Watson and Lof, 2004; Gersten \& Brengelman, 1996). What constitutes the 'it works' reasoning is as yet unclear. It may reflect the eclectic approach to treatment identified in many SLT studies (Joffe \& Pring, 2008; Rosseau, Packman, Onslow, Robinson \& Harrison, 2002; Roulstone \& Wren, 2001). As Roulstone (1997) advises, making explicit the thought processes of clinicians is necessary if the reasons underlying clinical decisions and actions are to be understood. This study of SLTs practising in Ireland aimed to examine what actually constitutes the 
reasoning provided by SLTs for treatment choices and whether science plays a part in those decisions.

\section{Method}

Design

This study, based in Ireland, used a mixed methods approach and consisted of two datacollection phases which explored clinical reasoning. Phase 1 was the quantitative component and used an electronic survey. This paper reports on Phase 2 which examined attitudes underpinning treatment choices and the therapy process. Ethics approval was provided by the University Research Ethics Committee

\section{Sampling and recruitment}

SLTs were recruited from community, hospital, and disability work settings via SLT managers who acted as gatekeepers. The managers were accessed via the University Practice Educator Database. This ensured that researcher was inviting participation from across all regions in Ireland. The researcher emailed an information sheet to the managers asking them to consider volunteering their department's participation, subsequent to agreement by their SLT staff. Seven departments volunteered and selection was based on the first three volunteering respondent groups representing community,hospital, and disability work settings.

\section{Method: Focus groups}

Focus groups were chosen as the vehicle for data collection to obtain a range of opinions and therefore articulate the collective view. Three focus groups were planned one in each of the following settings: community, hospital and disability work settings. The researcher travelled to each locality to undertake the focus group to increase the convenience for the participants. Although focus groups are said to work best with between 7-12 participants, no limit was set on participant numbers within the focus groups because the range of staff within individual departments varied considerably. However, the researcher aimed to recruit between 30-40 participants. Participants were consented prior to the commencement of the focus group. The focus groups were audio-recorded

\section{Stimulus questions}

Questions were developed based on the original research aims and from the outcomes of Phase 1 survey data. Therefore the focus group was constructed around developing meaning in relation to four key domains: client suitability, clinical experience, training and the role of science. The survey findings showed that client suitability was the primary reason for using therapies and the second reason for not using therapies. Clinical experience was the second most commonly used reason for using therapies. Being untrained was the primary reason for not using therapies. The role of science was included as this was central to the research question. 


\section{Data analysis}

Data were transcribed, anonymised and analysed using thematic analysis (Saldana 2009). An initial iterative analysis of each focus group identified preliminary codes and then themes. Coding took place over eight phases in total starting with nodding. Cross-group analysis revealed the extent to which the themes recurred across groups and eventually themes were identified and named. To determine coding reliability, as recommended by Anderson and Felsenfeld (2003), a research colleague was provided with the main thematic categories and quotes which were randomly selected from each category by a third party. This colleague was asked to place each quote into the thematic category felt to be most appropriate. Inter-rater agreement was $91 \%$. Participants were allocated pseudonyms and these have been used in reporting the findings.

\section{Findings}

Sample

In total 48 participants took part in the focus groups, all but 2 were females. The disability group was from the South region. The other groups represented two Eastern regions. The majority of participants represented senior grades (64\%), paediatric populations (69\%), were clinically-based (94\%) and had basic professional qualifications (81\%). Two-fifths (41\%) of participants had fewer than 5 years' experience while just over a quarter $(27 \%)$ has more than 15 years' experience.

\section{Overview of themes}

The themes emerging from the data reflect the complexities and paradoxes of clinical practice as described by SLTs. SLTs treatment decisions were often routine and at the same time thought through and whilst they involve patients, their decisions were not necessarily influenced by them. Although the SLTs appreciated research, they did not necessarily use research to inform their practice. Their practice was biased towards neither art nor science.

Decisions were scaffolded primarily on practice-evidence. The uniqueness of each patient results in dynamic and pragmatic practice, constraining the application of unmodified therapies and rule-based treatments. Three themes were identified: practice imperfect; practice as grounded and growing; and critical practice (see Table 1 for themes and sub-themes).

Table 1: Overview of themes and sub-themes

\begin{tabular}{|c|c|}
\hline Practice imperfect & $\begin{array}{l}\text { - Different patients, populations and therapists } \\
\text { - Uncertainty \& eclecticism }\end{array}$ \\
\hline $\begin{array}{l}\text { Practice as grounded and } \\
\text { growing }\end{array}$ & $\begin{array}{l}\text { - } \\
\text { - } \\
\text { - Casual and targeted learning }\end{array}$ \\
\hline
\end{tabular}




\begin{tabular}{|l|ll|}
\hline Critical practice & $\bullet$ & Pragmatism and reasoned learning \\
& $\bullet$ & Science and value \\
\hline
\end{tabular}

\section{Practice imperfect}

Practice imperfect encompasses the notion that treatment is not a recipe that a clinician can routinely follow to produce a perfect intervention episode. Equally, intervention is a mix of unique patients and eclectic therapists working in uncertain conditions.

\section{Different patients, populations and therapists}

The idea of imperfect practice revolves predominantly around its human elements. Many of the participants constructed treatment as 'individual packages' (Lizzie) where 'no one size fits all' (Matt). Treatment choices were shaped by patient characteristics (e.g. cognitive ability and medical issues) entailing 'a very symptomatic approach' (Leah) with SLTs 'constantly evaluating' (Niamh) how patients respond. The broader context described focused on client populations with individual therapies deselected if they were considered not to be fit for the population being treated: Stephanie talked of discarding the Nuffield as 'it wasn't working for our client group' whilst Becca explained that: 'the Shaker was never appropriate for our population'.

The SLTs additionally talked of how variable practice also results from individual clinicians, as Oran explained:

'The way I might do it might be very different to the way Matt does it'.

Being different was seen as being satisfactory 'as long as you reach that goal' (Matt).

Differences were evident between the distinct work settings with acute settings perceived as being more scientific: Cleena noted opportunities in hospital settings to be able to 'scientifically evaluate that a lot more clearly' with Jess noting that a hospital-based SLT could:

'define themselves more scientific because they're working in medical scenarios' (Jess).

\section{Uncertainty \& Eclecticism}

The SLTs quite clearly indicated that practice is comprised of many variables which effect decision making:

We don't have recipes, we just have loads of ingredients. We put them together in the way that we want' (Niamh).

The consensus was that 'most therapists take an eclectic approach, they mix and match' (Rose), resisting "dictated" approaches (Clara) and resulting in interventions which are ' $a$ bit of this and a bit of that' (Stephanie) as 'there's very few off the shelf programmes' (Jess). Indeed SLTs appeared to be comfortable with this as they 'like to be flexible' (Maryanne) and the overriding view is that evidence-based interventions are for ideal rather than real situations: for, as Jackie explained, 'dream children really'. Eclecticism results in few therapies being completely discarded as Sara explained:

'I would use part of it, I can't think of anything I've completely dismissed' (Sara). 
SLTs further explained that adapting therapies increases 'as your clinical experience kicks in' (Julie). This eclecticism encompasses research meaning that intervention can be ' $a$ mixture of the practical and the scientific' (Cleena). This eclectic 'pick and mix' (Josie) approach needs to be considered within the context of clinical uncertainty that was described by many participants: Oran talked of how the differing responses by patients 'change the whole flow of it!' and as Cleena said:

'Sometimes you're not even 100\% sure what the diagnosis is yet' (Cleena).

Thus, in a situation of such uncertainty as Cat states, practice 'can't be scientific as it's not clear cut'.

Another area of uncertainty related to evidence of the efficacy of interventions and ways in which the outcomes of interventions could be measured. Participants acknowledged that intervention might be improved by 'thinking in terms of outcomes from the beginning' (Eabha) and there was acceptance that some SLTs 'don't document very well either' (Becca). However, SLTs working in disability and community settings proposed the distinction between quantifiable outcomes and making a difference, as Maryanne summed up:

'While a person's scores may not change...you actually make a difference to their lives'

$$
\text { (Maryanne). }
$$

Clinical uncertainty means 'it's impossible to control for all the variables' (Helen).

Participants were well aware that factors such as the therapeutic alliance could be playing a part, with May noting that:

'Sometimes there a danger that ....it's the therapeutic relationship that was doing it' (May). Uncertainty was also linked to therapeutic trends as therapies 'come into vogue and then they go out of vogue' (Niamh) and acknowledgement of the deficit of research-evidence which led Stephanie to reflect: 'If there isn't an evidence base what do you do?' (Stephanie).

$\underline{\text { Practice as grounded and growing }}$

This theme addresses issues which facilitate decision-making and the ways that clinicians add to their repertoire of knowledge and skills through ongoing primarily practice-based learning.

\section{Automaticity and toolkits}

Automaticity is partly represented by a clinician's individual toolkit or 'bag of tricks' (Niamh). SLTs start from a base of college learning and add to their unique toolkit over time. Additions to the toolkit vary by work-setting as different areas of practice have ' $a$ core group of therapies' (Tara). Interventions and practices are retained in a clinician's toolkit if they are known 'to work'. As Grace stated, 'a lot of stuff I do, I do because it works'. Knowing what works, contributes to the clinician's comfort; this, in turn, impacts upon retention within their toolkit. Oran exemplifies this when saying:

'I never felt comfortable using it so I didn't give it a chance to see if it worked' (Oran). 
The clinician's toolkits are instruments for structuring practice. While trial and error contributes to the content of the toolkit as:

'You can try something. It doesn't have to work. It's not a lost session- you've just put something else in the tool bag' (Eabha).

Toolkits also reduce experimental practice as Rose says: 'you'll use it, rather than bumbling around and missing the mark'. Thus, experience can result in a degree of automaticity in practice where things are done 'without thinking' (Lee) or when favoured approaches are automatically adopted, for example, Matt explained: 'I would tend to use postural techniques first'. This approach to practice has clear echoes of pattern recognition and Oran succinctly explained this:

'It's an unwritten database in your head. Intangible. You know what works, what doesn't, what's gotten you into trouble, what hasn't, what clients have liked, what they haven't'

(Oran).

Although the thinking underpinning this decision-making is not readily articulated, it is not without logical merit as it reflects what Lee calls 'constant online problem-solving'. As Moira emphasized, 'it doesn't mean it doesn't include scientific knowledge'. However, this approach may negatively influence critical engagement as while SLTs 'sometimes try to think about what's new', they also 'often go back to the bag of tricks' (Becca). This means there is potential to 'get stuck in a rut and do the same thing' (May). The reasoning supporting this reflects pragmatism and habit, as Becca notes: 'You become used to what you use, it's easy and fast and effective'.

Thus, therapies may be mechanically employed resulting in SLTs not 'specifically targeting anything' (Oran) and often 'assuming the evidence was there' (Olivia).

\section{Casual and targeted learning}

SLTs accumulate their repertoires mainly from casual learning as Grace exemplified:

'Most of my day to day stuff wouldn't have been ....influenced by training' (Grace). This was a common phenomena as SLTs 'just go and do it' (Oran) and 'give it a go' (Siobhan). Formal learning also resulted in casual use once trained, as SLTs are 'in a position to be flexible... around it' (Cat). Thus, the SLTs evaluate the merits of their learning by undertaking training and then 'deciding myself' (Katie), primarily by 'reflecting on it afterwards and discussing it' (Lee). This active, experiential approach to learning naturally produces errors that in turn contribute to learning and from which 'you go forward' (Susie).

Colleagues are an 'invaluable' (Susie) source of learning meaning that SLTs are 'not lone rangers' (Ash). Learning from colleagues can take many forms including 'shadowing' (Olivia), 'talking' (Maggie), 'demonstration' (Oran) and 'pulling experience from others' (Ciara). Co-operative growth usually involves seeking the opinion of experts who have 'got a lot of experience' (Ella) and as Lee explained:

'If there's a stammering child everyone heads to Alice's office who's expert' (Lee) Contemplated action tends to follow on from that expert opinion, with SLTs 'taking from her experience and adapting it' (Siobhan). Students and new graduates are also a source of 
up-to-date knowledge especially as they can 'bring in an idea' (Ruth). SLTs actively pursued colleague's experience for a number of reasons including preventing 'tunnel vision' (Beth) and because it has 'more impact maybe than lots of stuff you read' (Pamela). It is prized for its efficiency and practicality:

'It's much quicker to access somebody who's next door than it is to read an article' (Lee).

\section{Critical practice}

Critical practice reflects that the ways in which SLTs indicated that they are critical thinkers who were sceptical about both non-scientific practices and EBP and demonstrating scientific behaviours beyond those related to research evidence.

\section{Pragmatism and reasoned learning}

SLTs' pragmatic approach takes many forms including a concern for functionality.

Treatment decisions therefore favour:

'Keeping it functional to the child's needs rather than following a set programme' (Beth). Functionality includes adapting therapies 'because of very different needs' (Beth) implying intervention may be less about programmes and evidence than practical outcomes. As Grace admits, 'I do it because it works. I wouldn't have checked the evidence behind it'. Research-evidence is criticised as being more suited to 'ideal conditions that real clinical situations' (Jess). Moreover, SLTs complain about the paucity of research-evidence often despite their best efforts to locate it. Stephanie explained that 'we've trawled the journals looking for evidence and it isn't there'. Concerns extend to 'conflicting evidence' (Cleena) and lack of clear direction as 'papers at the end of it say....further research' (Leah). While 'quantitative stuff doesn't tell you very much and you can't transfer it' (Lee), case-study research is it would:

'...be much more useful to read a very detailed case study about somebody so you have a real picture, something useful to take away that you might be able to apply to a similar client' (Lee).

For some therapies, as Beth says 'you can't' use them unless trained and Siobhan acknowledged that:

'If I hadn't gone on the course I wouldn't even attempt it' (Siobhan).

Formal training is especially important for 'invasive therapies like...DPNS' (Matt) although this can result in a pervasive feeling that 'now I can't do any harm because I've been trained' (Leah). The reasoning for undertaking such training has scientific undertones with SLTs acknowledging the need to understand the 'core concept' (Cat), the 'principles' (Niamh), or the:

'essence of it, why it's working, how important it is to do some parts exactly as designed' (Ash).

This knowledge enables SLTs to make informed treatment choices and builds assurance; a sentiment echoed by all participants:

'We don't feel confident in the therapy unless we've been on a training programme' (Matt). 
So training can be considered as a measured exercise, undertaken for learning 'a specific skill and where you need to be certified' (Oran).

\section{Science and value}

The SLTs expressed conflicting opinions with regard to research evidence. In some situations research evidence can influence change, with some practices being rejected and not used 'anymore' (Ella). Cat was clearly appreciative of more recent research saying:

'Research is coming out now and that's wonderful, it's becoming more scientific' (Cat). However, research is also rebuffed; Niamh explained that SLTs 'don't have to take it as gospel' and Leah seeing contradictions with experience as:

'This paper comes out that says it doesn't work at all. You might kind of go well I've ten other patients that say otherwise' (Leah).

The reasoning behind this includes 'evidence that shows both sides' (Leah), 'crap articles' (Oran) and 'that kind of research doesn't really speak to what we do' (Caro). SLTs also feel evidence is open to abuse particularly in relation to commercialised therapies with SLTs questioning 'how valid is their research' (Tara) and suggesting some therapies are 'designed to make people feel they are evidence-based' (Matt).

Despite these issues, SLTs also use research to support their concerns about some therapies:

'I never believed in it, and, then when the research came out I was delighted' (Lee). This conflict is expressed in a 'science but' argument. Annie sums this up by portraying clinical practice as 'science but not exact science'. So although numerous clinicians agree that 'to say speech therapy is art is doing your profession a disservice' (Maggie), they also are 'reluctant' (Annie) to be called scientists as: 'It's not science in the way that medicine is science' (Lee). This is reflected in feelings of being pressured by EBP, evident in terms such as 'squashed' (Lorcan), 'pushed' (Ruth) and 'shoehorned' (Ella). SLTs signalled scientific practice in other ways including the use of measurement which can function to support use of unvalidated therapies: that is, the use of unvalidated therapies is okay 'as long as you're evaluating it' (Oran). Experimentation is also identified:

'We are experimenting with approaches, evaluating outcomes and modifying them' (Leah). Thus, SLTs are 'problem solvers' (Sue) 'looking for different reasons, different causes or solutions' (Lizzie).

Scientific practice is also demonstrated in questioning including 'finding out where ideas come from ' (Marie) and asking what impacts outcome as Helen explains:

'Is it the method that's working or is it something about the way that I'm doing it?' (Helen). Scientific practice centres on 'critical thinking' (Caro), suggesting that SLTs are not 'blindly doing something' (Oran). Scepticism appears pervasive about therapies, especially those which may be well-packaged or 'very strongly commercialised' (Siobhan). It extends to therapies which 'factor in training' (Niamh) which is often seen as 'complete money making' (Julie). Scepticism was most often linked to 'anything with huge promises and a lot of money' (Jess). 


\section{Discussion}

The themes - practice imperfect, practice as grounded and growing, and critical practice will be considered under four constructs.

\section{A different arena}

Conture (1997:240) has referred to intervention as 'a problem-solving, manoeuvreaccording-to-circumstances approach'. This reflects SLTs views and mirrors the swampy lowlands described by Schon (1983). Decision-making is scaffolded on both the group (population) and individual characteristics of each singular patient and the group (discipline) and individual experiences of each clinician. This makes practice both routine and dynamic with each individual intervention episode being subject to multiple, unique and sequential decisions in line with theories of hypothetico-deductive reasoning (Higgs \& Jones, 2008). The implication is that treatment decisions are less about scientific notions of research-evidence and more about scientific thinking and behaviours. Essentially, it implies that rule-based interventions do not fit seamlessly with clinical practice. The sentiments expressed echo Firensuoli's (2000) argument, that there is an identified difference between what research does and what clinical practice is.

Pivoting practice on individual patients results in clinical uncertainty, the recognition and mastering of which is an important skill (Kitson, 1999). SLTs do not choose to ignore uncertainty but to decrease it in a variety of ways including via knowledge of the client group, toolkits, colleague opinion and scientific behaviours such as trial and error. Such attempts to reduce clinical uncertainty can be said to be evidence of critical practice, requiring clinicians be continually mentally-engaged. Practice is full of contradictions which contribute to this uncertainty. SLTs feel pressured by EBP but may use research to undermine therapies. Treatment is individualised but also dependent on population-specific knowledge. Interventions are scaffolded by toolkits but also eclectic with SLTs adopting a pick and mix approach to therapies in the way Hayhow (2010) has suggested. SLTs value measurement as a tool but reference immeasurable practice. Such opposites may however work in harmony with clinical reasoning being 'both simple and complex' (Higgs \& Jones, 2008:4). Population knowledge and toolkits for example, serve the needs of simpler practice and effectively underpin the individuation of practice and responding to complex patients. Measurement makes the use of unvalidated therapies more scientific.

The push towards use of evidence-based practices, rather than reducing clinical uncertainty as Gambrill (2005) suggests, may actually contribute to it and is partly interpreted as pressure. Barrier studies on the use of research-evidence (e.g. Nail-Chiwetalu \& BernsteinRatner, 2007) consistently highlight failures of uptake despite overwhelmingly positive attitudes to research (Dowsell, Harrison and Wright 2001). It is appropriate to ask if the EBP model, specifically the emphasis on research-evidence reflects the actualities of practice. Certainly, SLTs express appreciation for a variety of case-based data, perhaps because these data are more effective in moderating uncertainty as seen Firensuoli's (2005) work. 


\section{The authority of practice-evidence}

Clinical experience emerges as a primary foundation for treatment decisions and is articulated in a variety of ways and essentially represents an understanding of 'what works' (Kahmi, 1999:93). The underlying tone of conflict within participant discussions was less noticeable with regard to practice-evidence. Intervention which is highly populationspecific can be said to be composed of two core constructs: that which is accumulated and habitual and; that which is experimental and dynamic. SLTs toolkits are, in effect, what Heffernan (2011) calls pragmatic shortcuts to efficient practice and incorporate decision making represented by models such as illness scripts (Edwards et al., 2004) and 'rules of thumb' (Andre, Borgquist, Foldevi and Molstad, 2002). Through accumulated experience the clinician recognises similar features in cases by accessing stored knowledge which enables them to perform more efficiently. The notion of toolkits fits with the contention that the more experienced a clinician gets, the less logical their decision making processes are shown to be (Greenhalgh, 2002). However, the accumulated repertoire is heavily experimentally derived from clinicians' own experience, as well as that of colleagues' experience. The repertoire is not applied to individual patients in an inflexible or uncritical way. Toolkits include knowledge gained from making mistakes, which Lutterman (2011:7) call 'nuggets of gold', a natural consequence of the trial and error nature of practice. While Thompson et al. (2002) have argued that experience can provide a false sense of certainty and is of little help when facing new situations, SLTs willingness and tendency to experiment and problem-solve undermine this concern.

There is clearly a culture of looking to the group seen in numerous studies (e.g. MacKenzie et al., 2010) to check the validity of practice. This can also result in the rationalisation of poor practices although the scenario may not be so bleak as first assumed as scientific thinking is clearly present. Use of a new therapy, for example, appears contemplated and measured. Training is not automatically applied but reflected on. Colleague opinion is weighed in the context of the SLTs' own experience. Even the act of seeking out other opinion, of searching for clarity, of criticism and scepticism, of altering behaviour in a reasonable way represents scientific behaviour. While the reliance on such practiceevidence may be perceived as bad for the profession, this can only be bad if practiceevidence is wholly constructed as fallible, ungrounded and biased. There is no reason to suggest that this is, in fact, the case.

\section{The pivotal patient}

Despite toolkits, treatment choices are tailored for each patient; the SLTs agreeing with Gabbay and LeMay (2004) that there are no intervention cookbooks. The 'pivotal patient' is evident throughout all themes; reflecting the SLTs resistance to reducing patients to 'static, linear and simplistic labels' (Fourie, 2011:11). The 'patient as pivotal' explains why experimentation is required, why case-based research is so valued Rappolt (2003), why colleagues' expertise is targeted and why treatment decisions are under-influenced by external scaffolds. It is the case as Greenhalgh (2002:397) argues that the singularity of 
each individual client 'precludes any purely rule-based methods for.... selecting treatments'. Placing clients centrally in treatment decisions results in a functional approach that allows SLTs to adapt therapies, be flexible with rule-based treatments and form maximum intervention relevance for their patients. Evidence that functional goals can lead to good outcomes (see King et al.1998) suggests not just practicality but rationality in choosing this path.

The singular patient does have a broader context defined by the client group that patient represents, and the clinician's experience with that group. The client group influences routinised treatment selections suggesting that 'pattern recognition' (Higgs \& Titchens, 2000 ) is core to treatment decisions. Furthermore, the pivotal patient is largely defined in terms of pathologies rather than their preferences or values; that these appear to form a minor component of decision-making has implications for EBP. It suggests that the attempts to incorporate patient preferences within the model may have missed the mark. The SLTs appeared to favour a more medical model, lending support to the argument that hypothetico-deductive reasoning 'remains the most enduring clinical reasoning model' in practice (Edwards et al. 2004:314). In this model, tentative hypotheses are generated from patient cues on a continual basis. It points to treatment as an exercise which requires the SLTs' engagement and responsivity, heightening demands on them to be actively involved in the process.

\section{Science but...}

While SLTs appear reluctant to commit to 'being scientists' this may reflect the limits to an understanding of science-in-practice and the equating of science with research-evidence. This aligns with Kahmi's (2004:111) suggestion that science, truth and logic have little impact on SLTs professional identity. Lum's (2002:137) contention however, that 'to be a scientist, is in essence to think critically' would suggest that SLTs clearly do identify and execute scientific behaviours. The SLTs descriptions of 'trial and error' are similar to Logemann's (2004:134) proposition that much of what SLTs do is 'an individual clinical trial'. If science is systematic knowledge derived from experimentation (Federspil \& Vettor, 2000) then the SLTs practice may be said to be - at least in part - scientific. There are however, other behaviours which suggest a less than scientific approach, for example, affiliations with anecdotal sources, use of unvalidated approaches and automaticity. When accompanied by scientific behaviours such as trial and error and a questioning attitude they are less of a concern. Habitual practice for example, may have logical foundations. The seeking of other opinion reflects a search for solutions and clarity and open rather than dogmatic practice. This type of responsiveness is clearly defined by Adler (1987) as critical behaviour. Further, some attitudes may reflect practicality rather than a lack of rigour. The use of unvalidated treatments and rejection of some research for example, may be more to do with being pragmatic and valuing relevance and less a dismissal of science. It is clear as Kahmi (1999:94) has argued that SLTs are indeed 'not naive research consumers'. The concerns expressed by the SLTs echo previous findings for why research evidence in not routinely used such as conflicting evidence (e.g. Mullen, 
2005), lack of evidence (e.g. Law et al., 2004) and poor generalizability (Metcalfe et al., 2001). Certainly, the scepticism displayed about research-evidence signals scientific thinking and suggests that any reluctance to embrace EBP may be more logically founded than the literature might suggest.

Quite clearly the SLTs demonstrated a scientific attitude through their commitment to critical practice. Therefore, despite their standpoint as reluctant scientists, SLTs position themselves as what Bernstein-Ratner (2006) described as appreciators of science. Rather than being dictated to by research-evidence as arguably emphasized in the EBP model, the science of practice is characterised by clinicians operating in dynamic contexts using both scientific and less-scientific behaviours.

\section{Conclusions}

These findings paint both a complementary and contradictory picture of decision-making as constructed by SLTs. Essentially, intervention is not a textbook occupation; it is continually shifting although grounded in previous learning, and requires flexibility in operation. Practice is pivoted on both the patient and clinician, through their membership of groups and as individuals. The findings suggest that scientific thinking is a component of decision-making; a tool with which to approach the various ingredients and the dynamic nature of clinical practice. However, these scientific elements do not necessarily reflect EBP as typically constructed. Treatment decisions are primarily supported by practice evidence with research evidence and patient evidence - two of the pillars of EBP - underrepresented in the SLTs decision-making. Newbold et al. (2008) have expressed fears that not using research evidence makes the health professions more technical occupations than scientifically grounded professions. However, the opposite argument can also be made, that applying research-evidence to clinical practice may itself be a technical operation devoid of an understanding of the realities of practice. The findings support Oliver et al.'s (2014) argument that research should contribute to practice but should neither define nor lead it. Arguments which consistently place the breakdown for research use at the clinical rather than philosophical, theoretical or model level miss the point (McCurtin, 2012); currently practice is not well served by EBP and is deserving of models which better represent it.

\section{Recommendations}

1. The call for the literature to include more case-based research should be heeded if clinicians are to appreciate and utilise research and if research is to be seen as relevant.

2. Rather than ask how research evidence can be made more influential and trying to map unrepresentative models onto clinical practice, more research into the nature of practice is warranted, and models which better represent the complexity of clinical decision-making are required.

3. More attention needs to be paid to the role of the patient as partner in both research and practice. 